\title{
Applying refinement to the use of mice and rats in rheumatoid arthritis research
}

\author{
Penny Hawkins ${ }^{1}$ (1) $\cdot$ Rachel Armstrong ${ }^{2}$ Tania Boden ${ }^{3} \cdot$ Paul Garside $^{4}$. \\ Katherine Knight $^{5} \cdot$ Elliot Lilley $^{1} \cdot$ Michael Seed $^{6} \cdot$ Michael Wilkinson $^{7}$. \\ Richard O. Williams ${ }^{8}$
}

Received: 22 May 2015/Accepted: 24 June 2015/Published online: 14 July 2015

(C) The Author(s) 2015. This article is published with open access at Springerlink.com

\begin{abstract}
Rheumatoid arthritis (RA) is a painful, chronic disorder and there is currently an unmet need for effective therapies that will benefit a wide range of patients. The research and development process for therapies and treatments currently involves in vivo studies, which have the potential to cause discomfort, pain or distress. This Working Group report focuses on identifying causes of suffering within commonly used mouse and rat 'models' of RA, describing practical refinements to help reduce suffering and improve welfare without compromising the scientific objectives. The report also discusses other, relevant topics including identifying and minimising sources of variation within in vivo RA
\end{abstract}

All authors have contributed equally to this work.

Penny Hawkins

penny.hawkins@rspca.org.uk

1 Research Animals Department, RSPCA, Wilberforce Way, Southwater, West Sussex RH13 9RS, UK

2 Huntingdon Research Centre, Woolley Road, Alconbury, Cambridgeshire PE28 4HS, UK

3 UCB Celltech, 208, Bath Road, Slough SL1 3WE, UK

4 Institute of Infection, Immunity and Inflammation, College of Medical, Veterinary and Life Science, University of Glasgow, Sir Graeme Davies Building, 120, University Place, Glasgow G12 8TA, Scotland, UK

5 Home Office Animals in Science Regulation Unit, 2, Marsham Street, London SW1P 4DF, UK

6 Medicines Research Group, University of East London, Romford Road, London E15 4LZ, UK

7 Biological Services, Veterinary Research Facility, University of Glasgow, 464, Bearsden Road, Glasgow G61 1QH, Scotland, UK

8 Kennedy Institute of Rheumatology, University of Oxford, Roosevelt Drive, Headington, Oxford OX4 7FY, UK studies, the potential to provide pain relief including analgesia, welfare assessment, humane endpoints, reporting standards and the potential to replace animals in RA research.

Keywords Rheumatoid arthritis - Animal model ·

Refinement - Three Rs - Ethical review .

Humane endpoint · Welfare assessment .

Experimental design $\cdot$ Autoimmune disease

\section{Introduction}

Rheumatoid arthritis (RA) is a painful, chronic autoimmune disorder. Current treatments include non-steroidal anti-inflammatory drugs (NSAIDs), disease-modifying anti-rheumatic drugs (DMARDs) and biologics, but none are curative and there is a significant 'non-responder' rate (Strand et al. 2007; Julià et al. 2009). There is thus a need to develop more effective treatments for RA. In vivo studies, using animal 'models' of RA, are currently part of the research and development process for new or improved therapies and treatments.

However, procedures used to induce arthritis in animals can cause suffering, which may be mild, moderate or severe, depending upon the model and the duration of the study. Implementation of the three Rs (replacement, reduction and refinement) is thus a priority.

This document complements the literature on good practice within RA research by providing practical information on refinement that is often not included in publications. It is intended for a wide audience; researchers, animal technologists, ethics or animal care and use committees, veterinarians, funding bodies, regulators and anyone designing or reviewing studies involving in vivo models of arthritis worldwide. 


\section{Selecting the most appropriate approach and 'model'}

As a starting point, it is clearly essential to fully consider the mechanistic applicability and translatability of the various in silico, in vitro, in vivo and clinical methodologies used to address scientific questions relating to RA. Replacing or avoiding animal use should be a principal goal, as required in many legislations including European Directive 2010/63/EU (European Commission 2010).

For example, developments in three-dimensional tissue modelling show promise for in vitro drug evaluation, including in anti-inflammation research (Peck and Wang 2013), and in vitro cultures of bovine and human chondrocytes and cartilage discs are already used to investigate mechanisms of cartilage destruction in RA (Neidhart et al. 2000; Pretzel et al. 2009). Synoviocyte models using cells obtained from human RA or osteoarthritis patients during surgery have also been developed as test systems for candidate therapeutics (Smolian et al. 2001; Ribel-Madsen et al. 2012). It is important that use of human material is maximised - especially as fewer replacement surgeries are conducted in RA because patients are treated more successfully with DMARDs and biologics. Biologics with specific and well-defined molecular targets have also led to opportunities to replace animal RA models; e.g., analysis of immune and inflammatory parameters in peripheral blood mononuclear cells from patients before and after biologic therapy can reduce the need for animal use in some cases.

It is good practice to keep up with progress in such techniques, which can help to replace or avoid animal use, for example by screening out compounds without therapeutic benefit. Databases of in vitro, epidemiological and in silico models can help with this, e.g., http://www.go3r.org.

If there is no scientifically viable alternative approach, the choice of animal model should be guided by animal welfare considerations, aiming to minimise suffering in addition to the scientific purpose. ${ }^{1}$ There are useful reviews of animal models of RA in the literature (Bevaart et al. 2010; Patel et al. 2010; Bolon et al. 2011; Kollias et al. 2011; Vincent et al. 2012). Readers are also referred to initiatives such as 'Be The Cure' (BTCURE), a panEuropean research program to develop new RA therapies (www.btcure.eu). One of its aims is to develop an infrastructure to standardise procedures for generating and interpreting commonly used RA animal models.

These can be broadly divided into those that are (i) spontaneous, including mutant and genetically altered

\footnotetext{
${ }^{1}$ Note that in vitro work can also raise ethical and welfare issues, e.g.,, if primary cell lines are generated or animal serum used for cell culture.
}

strains, and (ii) induced. Spontaneous models progress naturally and generally involve a non-resolving, chronic condition. Induced models may be polyarthritic (involving a systemic response), which are more likely to be severe, or monoarthritic (induced by local challenge into a joint).

Species used in RA studies worldwide include non-human primates (marmosets and macaques), mice, rats, rabbits, zebrafish, pigs and dogs, but this report addresses mice and rats as they are most commonly used.

\section{Scientific issues}

There is some debate about the optimum in vivo RA models for particular disease aspects, as well as their translatability to human disease, with different models possessing different mechanistic and clinical features (Vincent et al. 2012). If animal models fail, it may be with respect to clinical predictivity, or to misapplication of the type of validity required, be this 'face validity' (similarity to the human disease features of interest), 'construct validity' (similar underlying biological mechanisms) or 'predictive validity' (whether there is a similar response to clinically effective therapeutic agents) (McGonigle and Ruggeri 2014). For further explanation and discussion of causes of reduced external validity, see van der Worp et al. (2010). A 'pathogenesis map' for RA to assist with the decision-making process regarding validity is set out in Vincent et al. (2012).

\section{Ethical and animal welfare issues}

A 'harm-benefit assessment', in which the potential harms to animals (i.e., pain, suffering or distress) are considered against the possible benefits of each project, is commonly used by regulators and ethics committees to make decisions about the justification for animal use (e.g., European Commission 2010; National Research Council 2011). The project should also have realistic objectives that are deliverable in practice.

With respect to identifying harms, factors to consider include:

- whether arthritis is spontaneous or an inducer is necessary;

- any need to boost or synchronise;

- number and frequency of interventions including anaesthesia;

- latency to onset of severe outcomes and their subsequent duration;

- number of joints affected;

- maximum level of suffering experienced by the animal;

- lifetime suffering experienced by the animal;

- potential to provide analgesia;

- numbers of animals required. 
The Group considered the welfare impact of poly- and monoarthritic models, with respect to severity and the number of joints affected. The consensus was that some of the classical polyarthritic models (e.g., adjuvant- or collagen-induced models) generally result in a more severe arthritis and so should only be used as translational tools if there is strong supporting evidence of a relevant disease mechanism. Low severity (as opposed to hyperalgesia) monoarthritic models, including zymosan-induced and antigen-induced arthritis (Patel et al. 2010; Vincent et al. 2012), possess many relevant disease processes and, being less severe, should be used instead of polyarthritic models wherever possible. In addition, if only one limb is affected then the animal is able to compensate by redistributing weight between the other three limbs.

Harm-benefit assessments may be more complex for novel models that are less well established. Some may be more severe, e.g., the SKG mouse which develops severe arthritis plus extra-articular inflammation (Yoshitomi et al. 2005), while others can be manipulated to have reduced severity with greater clinical relevance (e.g., the $\mathrm{KBxN}$ serum transfer model; Montero-Melendez et al. 2011).

\section{Sources of variation in disease development and progression}

A number of factors can lead to variations in the incidence, severity and timing of arthritis, which can affect the number of animals needed to obtain a statistically significant result. Potential sources of variation, and actions that can be taken to minimise these, are set out in Table 1.

\section{Refinement of animal models}

Once the most appropriate model has been chosen, the next step is to ensure that refinement is fully implemented. An effective approach is to set out the whole life experience of the animal and consider how each potentially painful or distressing event could be refined, collectively leading to a significant overall reduction in suffering (European Commission 2012).

Table 2 sets out possible adverse effects within RA studies, with potential ways of ameliorating these. Supplementary text to explain some of the entries is set out below.

\section{Housing and care refinements}

Husbandry refinements, including appropriate environmental enrichment, benefit animal welfare and should be provided unless there is sound scientific justification to withhold them. However, it is essential to evaluate any effects on data variability (Mikkelsen et al. 2010) and to allow for these within the experimental design.

Based on the human experience of arthritis, affected animals should benefit from being able to keep warm and comfortable, exercise as appropriate and reach food and water easily. Temperature may be especially important for animals in RA studies, since healthy mice, given an opportunity to select their thermal environment, choose an ambient temperature of $30-31{ }^{\circ} \mathrm{C}$, considerably above the range in most facilities (Gaskill et al. 2009, 2012). This suggests that it is good practice to review ambient temperature levels for rodents in RA studies and provide a sufficient quality and quantity of nesting material. Besides the animal welfare implications, the systemic sympathetic response to cold stress can affect data quality in studies relating to immune function (Karp 2012; Kokolus et al. 2013), and it is worth considering how this might also apply to RA projects.

Regarding enrichment, shifting attention away from pain benefits human patients (Ulrich 1984; Chan et al. 2012; Havey et al. 2014), and distraction from pain also modulates pain perception in animals (Gentle and Tilston 1999; Ford et al. 2008). An appropriately stimulating environment will therefore likely help to improve welfare and reduce pain perception in animals on RA studies.

Standard principles for housing, husbandry and care of mice and rats used in RA studies are:

- Soft litter, to reduce pain on walking.

- Sufficient soft, non-tangling nesting material to keep comfortable, cushion sore joints and enable thermoregulation.

- An appropriate group of cagemates for social animals, depending on age, sex and strain.

- One or more refuges, to permit natural behaviour and alleviate potential anxiety in animals with compromised mobility.

- Effortless access to easy-to-eat food and water, to cater for disability.

- Appetising food, to counteract or prevent weight loss.

- Proactive welfare management as opposed to reactive. For example, animals should be acclimatised to cage provisions, appetising food and hydration agents before arthritis is induced.

Defined sources of enrichment items should be used, because contaminants (e.g., dioxin), present in some oils and bleaching agents, can act as confounds (e.g., by affecting Cyp1A1 gene activity; Tischkau and Mukai 2009). Standardisation can be managed in the same way as regulatory toxicology studies, in which in-house Quality Assurance groups set limits of acceptability for different substances in litter, nesting materials and enrichment items. These limits are used to review Certificates of Analysis that 
Table 1 Sources of variation in animal models of RA

\begin{tabular}{ll}
\hline Sources of variation & Ways of addressing these \\
\hline
\end{tabular}

Protocol

Variation in protocols for inducing arthritis

Different protocols for assessing outcomes, e.g., with respect to clinical assessment, welfare assessment or histopathology

Different protocols for assessing therapy efficacy, given that the relevance of the drug target depends on the underlying mechanism

Environmental disturbance due to husbandry, scientific procedures and observations, or maintenance/construction work

Statistical power

Variation in batches and quality control of biologicals such as collagen, lipopolysaccharide and Mycobacterium

Animals

Different species, strains, sexes or ages of animals

NB Some outbred strains are cheaper than inbred and can respond with arthritis-but due to genetic variability, severity can range from no response to extreme

Lack of proper colony management, leading to (i) genetic contamination (ii) incomplete inbreeding or (iii) genetic drift, resulting in unpredictable variations in susceptibility

Variations in health status, e.g., pinworm infection

\section{Environment}

The length of time that animals have spent in the facility; i.e., animals housed for longer before induction may be more susceptible to arthritis in the case of collagen models

Level of biocontainment, i.e., whether in individually ventilated cages (IVCs) or conventional microbial environment

Type of litter, nesting material, enrichment items and diet; interactions with humans
Search the literature for suitable standardised protocols ${ }^{\mathrm{a}}$ and consult colleagues

Monitor progress with initiatives such as BTCURE

Research accepted assessment methods, ensuring discriminative power (see below)

Adapt the histopathology score to the specific model

Research the appropriate efficacy measure for each target and define outcome-based assessment criteria for potential therapies, e.g., clinical scores or cellular responses

Keep noise to a minimum

Capture and handle animals with care

Minimise the number of technical acts (e.g., administration of substances and anaesthesia) — but not by increasing the impact on the animal (e.g., without excessive dose volumes)

Reduce husbandry and maintenance procedures, like cage cleaning and cleaning animal rooms, to the minimum necessary for good health

Find out when cages and rooms are cleaned and avoid conducting procedures immediately afterwards

Conduct power calculations, use appropriate numbers, and define appropriate statistical analysis at the project planning stage. Plan for 'dropouts'

Use pilot experiments, where appropriate, to define acceptable limits of severity and ensure statistical power

Only use defined and/or batch tested biologicals, ensuring they are in date

Search the literature to help select the appropriate animal—but be critical and do not simply follow tradition; research and review current approaches with respect to species, sex, strain and age

Use inbred strains to reduce variability and extremes in responses

Ensure good colony management; 'refresh' in-house colonies periodically by returning to founder stock; ensure frequent genotyping of generations

Consider establishing a colony for long term projects, but ensure overbreeding and wastage are minimised

Apply good health care and colony management, led by animal technologists and the attending veterinarian

See comments for 'variation in protocols for inducing arthritis' above

Be aware of model-specific pros and cons, e.g., mouse C57B16 CAIA seems to require IVC housing, but the SCW model may be more responsive in an open-top cage environment

Ensure that these are carefully selected and adequately described in publications (see Sect. 3) 
Table 1 continued

\begin{tabular}{|c|c|}
\hline Sources of variation & Ways of addressing these \\
\hline \multicolumn{2}{|l|}{ Operator effects } \\
\hline $\begin{array}{l}\text { Variations in performance of techniques, with respect to } \\
\text { expertise or level of awareness of correct protocol—an } \\
\text { establishment 'culture' issue }\end{array}$ & $\begin{array}{l}\text { Ensure that good practice is observed with training, } \\
\text { supervision, assessment of competence and Continuing } \\
\text { Professional Development, seeking advice both internally } \\
\text { and externally as necessary } \\
\text { Account for any variation in experimental design; block by } \\
\text { operators (personnel conducting each procedure) }\end{array}$ \\
\hline \multicolumn{2}{|l|}{ Other sources of variation } \\
\hline $\begin{array}{l}\text { Unexplained variation between different facilities using the } \\
\text { same protocols, sexes and strains }\end{array}$ & $\begin{array}{l}\text { Ensure good attention to detail at all steps when inducing } \\
\text { arthritis } \\
\text { Liaise with external colleagues and compare how protocols are } \\
\text { interpreted } \\
\text { Discuss the animals' experiences with animal technologists } \\
\text { and care staff to identify differences in husbandry protocols } \\
\text { Monitor and compare physical environments, e.g., noise, } \\
\text { temperature fluctuations } \\
\text { Conduct a full health screen of animals } \\
\text { Ensure that publications include an appropriate level of detail } \\
\text { to help interpret results if differences persist }\end{array}$ \\
\hline
\end{tabular}

a Standardised protocols can help to promote consistency, but should be critically considered every time. 'Standardised' does not always mean 'fit for purpose', and standard protocols may involve greater animal numbers or suffering than is desirable. An alternative approach to standardisation between facilities is for each to use models that enable good reproducibility with minimal suffering, and regular refinement, ensuring that protocols are written up in adequate detail. This approach also further disseminates information about good practice

accompany such materials. Describing the sources of all materials that are provided will help others to interpret the results and conclusions of publications (Hutchinson et al. 2005).

\section{Catching animals prior to handling}

It has been demonstrated that being caught by the tail is stressful for mice and induces anxiety (Hurst and West 2010; Gouveia and Hurst 2013). Avoiding capture by the tail in RA studies, by catching and restraining using cupped hands, tunnels or Vetbed ${ }^{\circledR}$, could thus decrease stress as well as reducing the risk of causing discomfort through involuntary extension/flexion of arthritic joints, or if animals have been injected close to the tail base.

\section{Arthritis inducers and their administration}

Local adverse effects due to inducers can be minimised by reducing the dose, volume and frequency of administration. The feasibility of this approach depends on the nature of the antigen, its solvent, and the adjuvant, and can be evaluated in pilot titration studies, using scoring systems to monitor the onset and severity of arthritis.

Regarding administration, wide gauge needles cause more pain and increase the risk of 'tracking', where a tunnel remains under the skin following withdrawal. The inducer can leak into this, causing irritation and further discomfort or pain. To avoid tracking, withdraw the needle slowly and smoothly, applying slight pressure with the thumb. Mycobacteria for adjuvant and collagen-induced arthritis should be ground very finely to prevent 'stacking' in finer needles (Brand et al. 2007) and consequent variability in incidence and response. NB Care must be taken when alternating between strains of Mycobacterium, as $M$. butericum can induce more severe rat adjuvant disease than M. tuberculosis.

Many protocols use intradermal injection, but this leads to a higher incidence of ulcers, especially if administered close to the tail. In the experience of Group members, subcutaneous injection at single or multiple sites further from the tail (e.g., on the flank) can reduce ulcer incidence while still reliably inducing arthritis. This may be possible in some studies that do not depend on intradermal injection, and a pilot study to evaluate an alternative administration protocol may be justifiable. If there is scientific justification for intradermal injection, sites should be chosen with care, taking into account both the animal's movements and areas that will be affected during restraint.

\section{Study duration}

Refinement can also be achieved by reducing the duration of experiments, provided this is compatible with the study aims, i.e., all the necessary data can be obtained within the study time. There may also be scientific reasons not to 
Table 2 Adverse effects and refinement

\begin{tabular}{ll}
\hline Potential adverse effect & How this may be refined
\end{tabular}

Administration of RA inducer

Capture, handling and restraint

Pain due to administration of inducer (intradermal or subcutaneous injection)

After effects of anaesthesia e.g., dehydration, inappetence

Pain or ulceration around injection site

Administration of inducer by intraperitoneal injection

Specific adverse effects due to adjuvant, e.g., granuloma, irritation, lesions

Effects of lipopolysaccharide 'boost' - may be 'shock'-like cytokine storm
Competent, empathetic capture and handling (e.g., capture by cupping or tunnel, not tail)

Habituation to handling and restraint

Use gaseous anaesthesia for intradermal routes, to reduce pain and increase accuracy

Inject intradermally into the rump, not the tail base. If the site is painful, capture or restraint by the tail will hurt

Keep volumes and doses to the minimum necessary; it is better to use multiple sites if larger volumes are needed-but not too close together as injectates can coalesce, causing granulomas

Ensure injectate has been formulated so as to minimise swelling and pain

Give treats such as Nutella ${ }^{\circledR}$ or sunflower seeds

Ensure animals can reach water or food with high water content such as wet mash, transgel, satsuma segments

Provide oral glucose or rehydration with saline if necessary

Monitor body mass and dehydration

Inject into the rump for less risk of ulceration; additional injections can be into the flank if needed

Never inject into the foot-this is too painful and not necessary

If animal pays attention to injection site, apply topical local anaesthetic and review anaesthesia and injection protocol

Ulceration should heal after 4 to 7 days-if not, implement a humane endpoint based on characteristics and persistence (see Sect. 7)

Consider needle gauge with care and avoid 'tracking'; implement humane endpoint if significant tracking

This is 'going blind' and adequate training is essential in order to avoid injecting into an organ or the gut. Never administer Freund's complete adjuvant (FCA) via this route

Use the least harmful adjuvant possible-monitor the literature for alternatives and challenge the use of problematic compounds like FCA

Shaving while under anaesthesia, immediately before injection, helps to monitor adverse effects if these are likely

Trial using incomplete Freund's adjuvant for a less severe reaction

Provide additional nutritional and hydration support for animals before injection

Define appropriate humane endpoints

Monitor body mass and dehydration, remove faecal plugs 
Table 2 continued

\begin{tabular}{|c|c|}
\hline Potential adverse effect & How this may be refined \\
\hline \multicolumn{2}{|l|}{ Effects of arthritis } \\
\hline \multirow[t]{7}{*}{$\begin{array}{l}\text { Painful joints, sore feet, lameness, disability and } \\
\text { distress }\end{array}$} & $\begin{array}{l}\text { For intra-articular induction protocols only induce in a } \\
\text { single joint }\end{array}$ \\
\hline & $\begin{array}{l}\text { Implement husbandry refinements, e.g., weighing boats } \\
\text { to sit in, refuges designed so that animals do not have } \\
\text { to turn around, long nozzles on drinking bottles, soft } \\
\text { sawdust litter, short and soft nesting material (long } \\
\text { strands can wrap around sore legs) }\end{array}$ \\
\hline & $\begin{array}{l}\text { Provide soft, appetising diet or diet gel (accustom } \\
\text { animals to this before the acute phase) }\end{array}$ \\
\hline & Pick up and handle using washed Vetbed ${ }^{\circledR}$ \\
\hline & Handle very gently and empathetically \\
\hline & Give analgesia if possible (see Sect. 5) \\
\hline & $\begin{array}{l}\text { Refine humane endpoints; include consideration of } \\
\text { study duration and the level of disease severity } \\
\text { necessary to answer the scientific question }\end{array}$ \\
\hline \multirow[t]{2}{*}{ Acute pain } & $\begin{array}{l}\text { Provide analgesia if possible, e.g., opioid during 'attack' } \\
\text { phase }\end{array}$ \\
\hline & $\begin{array}{l}\text { Provide appropriate environmental enrichment, and } \\
\text { group housing for social animals, to help shift } \\
\text { attention from acute pain }\end{array}$ \\
\hline \multicolumn{2}{|l|}{ Other welfare issues } \\
\hline \multirow[t]{5}{*}{ Behavioural problems, e.g., aggression } & $\begin{array}{l}\text { Question scientific justification and necessity for using } \\
\text { aggressive strains, or male mice of some strains e.g., } \\
\text { DBA1, C57BL/6 }\end{array}$ \\
\hline & Use littermates where possible \\
\hline & $\begin{array}{l}\text { Review husbandry with respect to group size and } \\
\text { number/design of refuges }\end{array}$ \\
\hline & $\begin{array}{l}\text { If single housing is necessary for welfare reasons, } \\
\text { ensure animals have adequate enrichment, especially } \\
\text { a refuge and plenty of nesting material }\end{array}$ \\
\hline & Remove aggressors if necessary \\
\hline $\begin{array}{l}\text { Inherently severe arthritis in particular models, } \\
\text { e.g., spontaneous SKG mouse, and species or } \\
\text { strains (e.g., Lewis or DA/Ola rat) }\end{array}$ & $\begin{array}{l}\text { Explore potential to answer the same question using a } \\
\text { less severe model, e.g., Methylated Bovine Serum } \\
\text { Albumin (mBSA) model, or a less susceptible strain }\end{array}$ \\
\hline
\end{tabular}

prolong studies, due to the risk that the disease will enter the 'repair phase', with associated periostitis or ankylosis that can confound the results.

\section{Adverse effects of RA models}

Some factors that require special attention are set out below.

\section{Boosting}

Boosting is not used in rats, but is sometimes used in mice to support antibody-induced collagen induced arthritis, or to synchronise the collagen-induced arthritis (CIA) model. Boosting commonly takes the form of an intraperitoneal injection of lipopolysaccharide (LPS) or subcutaneous administration of collagen in Freund's incomplete adjuvant (FIA); Freund's complete adjuvant (FCA) should not be used due to the challenge response to the adjuvant.

Administering LPS is stressful and induces cytokine release that can cause severe adverse effects including shock, diarrhoea, and malaise. Animals may not drink, and may develop faecal plugs that, coupled with diarrhoea, can be life-threatening if not checked regularly and cleaned away. However, there may be justification for using LPS if it allows study duration to be reduced and/or fewer animals used because the incidence of arthritis is reliably increased. Collagen/FIA boosting should be subcutaneous, and can be refined by restricting sensitisation to one side of the animal and administering the boost at a separate site. The choice of ligand should also take translatability and adverse effects into account; for example, the various TLR ligands may model different aspects of RA more effectively than more 
commonly used agents such as LPS or FIA, but may have different adverse effects.

\section{Acute phase of arthritis}

Animals experience the most severe pain during the acute or 'attack' phase, during which they need close monitoring and additional care, including analgesia if feasible (see Sect. 5). Most therapeutic studies are carried out during this acute phase and it is not usually necessary to extend them into the chronic, resolving phase (see below). The severity of the acute phase should be refined to provide the required statistical power for the primary outcome and not more.

\section{Resolving phase}

If polyarthritis models are taken into the 'chronic resolving' or 'recovery' phase (e.g., to study bone remodelling), the impact on the animal must be carefully considered. The consequences of periostitis become more prominent (especially in rat adjuvant arthritis) as aberrant bone outgrowths develop within the paws and along the bone shafts. Although acute inflammation may have receded, periostitis and spondyloarthropathies are painful and add significantly to the lifetime severity.

Joint damage can be so severe in polyarthritis models that resolution to normality is impossible. Low acute-load models should be used to investigate factors influencing resolution, as they allow mechanisms in joint resolution to be seen as well as causing less suffering (Montero-Melendez et al. 2011).

\section{Control groups}

In studies of potential therapeutics, animals in control groups that do not receive the candidate therapeutic agent will develop the most severe form of disease and are of special concern. The requirement, and humane endpoints, for controls should be very carefully considered. In some fields, controls can be avoided by using 'historic' controls from the literature or the same institution. Unfortunately, in RA studies this is likely to mislead due to variations between institutions, contemporaneous environmental factors, and the protocols used. However, sharing control groups from different, contemporary experiments within the same institution is valid, and should be encouraged, provided they possess sufficient power.

\section{Aggression}

Inappropriate enrichment can cause aggression in male mice (Marashi et al. 2003), and male DBA/1 and
C57BL/6 mice, both of which are used for experimental arthritis, are especially prone to aggression. However, the risk can be reduced by establishing groups early, using littermates, ensuring that animals are not subsequently mixed and selecting appropriately designed refuges. For mice, two refuges or dual entry/ exit designs can defuse aggression ( $\mathrm{T}$ Boden pers. comm.), but if it persists, it may be necessary to remove aggressor(s) since fighting may cause stress, injury and infection-which can all influence arthritis development. Aggression between arthritic rats is not expected and indicates a serious welfare issue that should lead to a review of husbandry and experimental protocols.

\section{Adjuvant arthritis}

Adjuvant arthritis models, such as intradermal/subcutaneous administration of CFA to rats, or pristane to rats and mice, can easily lead to severe outcomes. Severity may be reduced by refining the initiation doses, e.g., reducing the dose of pristane for pristane arthritis, without compromising the arthritis outcomes (Malik et al. 2011).

The severity of CFA arthritis in rats can be compounded by systemic disease that, if uncontrolled, includes liver granuloma (which can affect Cyp enzyme activity), skin disease, splenomegaly, ulceration at the injection site, tail lesions, eye disease and disuse of the hind paws leading to dragging ('sledging'), and ulceration of the hind feet.

Although there are welfare concerns, there may be scientific benefits to consider as part of a harm-benefit assessment. The 'structural validity' of CFA-induced arthritis is different to other models (Patel et al. 2010) with cell mediated immune responses predominating, which may benefit the scientific objectives of some studies. In addition, drug responses can be more easily distinguished (Bolon et al. 2011), and when inbred strains are used responses tend to have low variability, with robust incidence and very predictable disease onset. These points mean that numbers do not need to take variability into account, so can be kept lower than for murine models. Therefore, there may be scientific justification for using CFA, but this should be very closely scrutinised and every opportunity taken to reduce suffering; pilot studies may be advisable before using this model for the first time.

Older publications describe the injection of the inducer into one hind paw, inducing the 'primary response', a chronic granulomatous reaction. The 'secondary response' of inflammation in the contra-lateral paw reflects the systemic arthritis. This protocol is incapacitating, can lead to severe disease, and should not be used. 


\section{Arthritis in genetically altered (GA) strains}

Some GA strains require careful monitoring, as the severity of arthritis may be unexpectedly severe or chronic. For example, prostaglandin- $\mathrm{D}_{2}$ synthase knockout mice have an exaggerated delayed-type hypersensitivity response, which would be predicted to result in severe disease if expressed in collagen arthritis (Trivedi et al. 2006); indeed, $\mathrm{PGD}_{2}$ antagonism exacerbates disease (Maicas et al. 2012). Where exacerbation of disease is expected in a GA line, models or protocols of reduced severity, such as monoarticular or reduced inducer dose, should be used wherever possible.

\section{Analgesia in models of RA}

All arthritis models involve pain and some are in fact used as models of inflammatory pain (Colpaert 1987; Honoré et al. 1999). Some pain can be attributed to the acute inflammatory response, but chronic pain may also develop, with hyperalgesia associated with a variety of neurochemical changes in the spinal cord (Colpaert 1987; Honoré et al. 1999; Christianson et al. 2010; Bas et al. 2012). This is evidenced by the fact that rats and mice continue to self administer analgesics in the resolving phase, even though paw swelling has abated (Colpaert et al. 2001; Wooley et al. 1981). The goal of analgesia in RA studies is to minimise acute and chronic pain without having a significant negative impact on scientific validity.

\section{Analgesics and scientific validity}

Most analgesics affect the immune system in some fashion (Paska et al. 1986; Earl et al. 1994; Dinda et al. 2005; Pulichino et al. 2006), so there can be concern about providing analgesia for fear of introducing a confound. However, there is increasing evidence of effects upon many body systems (including immunity) of unrelieved pain and distress in animals, and how these can influence the experimental outcome (Baumans et al. 1994; Livingston and Chambers 2000; NHMRC 2008; Ren and Dubner 2010). It is also noteworthy that analgesics are rarely excluded from human clinical trials, which are therefore subject to the same confounding influences.

The authors therefore propose that analgesia should be used unless there is sound scientific justification otherwise, e.g., if it can be demonstrated that analgesia would make it difficult to attribute therapeutic effects to the study compound, or if a class of analgesic could affect unpredictably the disease severity in studies using GA animals. For a discussion of decision making regarding pain alleviation, see Carbone (2011).
Pain relief protocols can be designed to minimise potential impact on the scientific objectives. For example, analgesia can be tailored to periods when inflammation is at its peak and likely to be especially painful (Khachigian 2006; McCarthy et al. 2012), with administration in anticipation of (rather than in response to) the pain in order to increase efficacy.

\section{Potential analgesics for RA studies}

When considering whether to provide analgesia, two essential questions are (i) what effects occur at analgesic doses, and (ii) will these necessarily invalidate experimental outcomes? There are a number of reports of the use of analgesics in arthritis models without negative impacts on the experiment; some examples are set out below.

Gabapentin has been found to be effective in the attenuation of allodynia during the chronic phase of murine $\mathrm{K} / \mathrm{BxN}$ arthritis (Christianson et al. 2010) and has also been shown not to interfere with the immune response (Van Loo et al. 2006). Gabapentin, the NSAID ketorolac and the TNF receptor antagonist Etanercept $(\mathrm{R})$ have all been reported as effective during the acute phase, whereas gabapentin alone was effective on allodynia in the chronic phase (Christianson et al. 2010). Since NSAIDs are anti-inflammatory and can have DMARD activity (Seed and Burnet pers. comm.), anti-TNF is antirheumatic, and both are only effective in the acute phase, gabapentin could be used.

Buprenorphine, a partial agonist opiate, does not prevent the development of a reliable arthritic response in mice when administered in drinking water (M. Burnet, Synovo, pers. comm., see below), but neither direct comparisons with non-treated mice, nor effects on joint histopathology, have been assessed. Buprenorphine reduced spinal neuronal discharges and reduced allodynia during the acute and chronic phases of mouse CAIA (Bas et al. 2012). However, oral administration of buprenorphine at analgesic doses $(2 \mathrm{mg} / \mathrm{kg}$ twice daily) in rat SCW arthritis inhibited inflammation and joint erosion (Volker et al. 2000).

Paracetamol (acetaminophen) is potentially a suitable analgesic, since it possesses less anti-inflammatory activity than NSAIDs and COX-2 inhibitors. Paracetamol at $50 \mathrm{mg} / \mathrm{kg}$ significantly reduced nociceptive evoked and spontaneous spinal discharges in adjuvant arthritis (McQueen et al. 1991). It also reduced inflammatory hyperalgesia without affecting carrageenan inflammation and central hyperalgesia (Bianchi and Panerai 1996).

Therefore, it is possible to provide analgesia in some RA studies, and some institutions apply analgesia routinely. However, further research is needed into suitable analgesic regimes, including evaluations from the time of induction, or specifically the attack and chronic phases, to assess 
analgesic efficacy and dissociate these from arthritic outcomes. If in doubt, the veterinarian should be consulted, literature searched and pilot studies conducted if necessary.

\section{Pilot studies}

Pilot studies could establish whether analgesia might be provided at one or more stages of an RA study without significantly compromising the science. Although the animals in the full study will benefit if it is shown that pain relief is feasible, pilot studies to evaluate this will cause suffering and should undergo a harm-benefit assessment, with full implementation of the three Rs. Ideally, the pilot study should be designed such that data from it could potentially be incorporated into the main study, to avoid using 'extra' animals.

\section{Administration of analgesics}

There are three main options for analgesic administration; parenterally or orally by either gavage or self-administration. There are pros and cons associated with each of these routes (Table 3). For guidance on administering analgesia in nut paste (e.g., Nutella ${ }^{\circledR}$ ), see Jacobsen et al. (2011) and
Abelson et al. (2012). Others have reported providing buprenorphine in the usual diet (Molina-Cimadevila et al. 2014).

\section{Assessing animal wellbeing, pain, suffering or distress in RA studies}

Species and strains vary regarding susceptibility to arthritis and how pain-coping behaviour is expressed. An effective day-to-day welfare assessment system should therefore be tailored to the species, strain and protocol, with input from the researcher(s), animal technologists and the veterinarian (Hawkins et al. 2011; European Commission 2012, 2013). All those responsible for assessing animals should receive adequate training in recognising indicators of suffering associated with each project and in using the relevant recording systems.

Assessment often involves handling, and animals used in RA studies may be in pain, so they should be habituated to empathetic, careful capture and manipulation. For example, mice or rats with swollen paws should never be picked up from cage lids. However carefully it is done, handling animals during the attack phase is still likely to be

Table 3 Advantages and disadvantages of parenteral versus self-administration of analgesics in RA studies

\begin{tabular}{|c|c|c|}
\hline Method & Advantages & Disadvantages \\
\hline $\begin{array}{l}\text { Parenteral } \\
\quad \text { administration }\end{array}$ & $\begin{array}{l}\text { Can be reasonably certain that required } \\
\text { dose has been reliably delivered } \\
\text { New slow-release opioid preparations are } \\
\text { being developed which may offer } \\
\text { prolonged and sustained analgesia } \\
\text { without the need for re-dosing }\end{array}$ & $\begin{array}{l}\text { Capture, handling and restraint for administration may } \\
\text { be painful and stressful, especially in the attack phase } \\
\text { or if animals are in chronic pain } \\
\text { Painful injection, risk of infection, risk of reduced } \\
\text { disease on repeated administration } \\
\text { A 'standard' dose may not be appropriate for the } \\
\text { individual }\end{array}$ \\
\hline Orally by gavage & $\begin{array}{l}\text { Can be reasonably certain that required } \\
\text { dose has been reliably delivered } \\
\text { Does not require injection }\end{array}$ & $\begin{array}{l}\text { As for parenteral administration, plus gavage procedure } \\
\text { can be distressing } \\
\text { Some physical risk to animal, e.g., misdosing into } \\
\text { trachea, damaging oesophagus }\end{array}$ \\
\hline $\begin{array}{l}\text { Self-administered in } \\
\text { water }^{\mathrm{a}} \text { or diet }\end{array}$ & $\begin{array}{l}\text { No handling or restraint required } \\
\text { Involuntary self administration, or can } \\
\text { train animals to recognise water with } \\
\text { analgesic } \\
\text { Animals can dose themselves optimally } \\
\text { Cage intake easily measurable }\end{array}$ & $\begin{array}{l}\text { Individual intake unknown } \\
\text { Severe pain could reduce ability to access food or water } \\
\text { and thus analgesia } \\
\text { May be issues with absorption or bioavailability }\end{array}$ \\
\hline $\begin{array}{l}\text { Self-administered in } \\
\text { 'treat' food }\end{array}$ & $\begin{array}{l}\text { No handling or restraint required } \\
\text { Can accustom animals to taking treats } \\
\left(\text { e.g., jelly, Nutella }{ }^{\circledR}\right) \text { so that they will } \\
\text { readily self-administer } \\
\text { Animals can dose themselves optimally }\end{array}$ & $\begin{array}{l}\text { As for self-administration in water/diet } \\
\text { May not readily take treat with analgesic } \\
\text { Rate of intake can vary with several factors, e.g., } \\
\text { position in group hierarchy }\end{array}$ \\
\hline
\end{tabular}

a Example regimes; $240 \mathrm{mg}$ paracetamol in $140 \mathrm{~mL}$ drinking water, or $1 \mathrm{mg} / \mathrm{L}$ buprenorphine in $150 \mathrm{~mL}$ water per diem (T Boden; M Burnet, Synovo GmbH, pers. comm.) 
painful, so a compromise is needed between ensuring adequate assessment and minimising (or even avoiding) catching and handling. Some indicators can be assessed without handling, such as 'pain faces' (below). Note that mice and rats are nocturnal and most active during the dark phase, so important behavioural signs may be missed if animals are only observed when it is light.

The monitoring protocol will also depend upon the study phase, e.g., one author's facility assesses animals three times a day during the acute phase of CAIA following LPS administration. If 'rescue analgesia' has been agreed, daily full assessment (involving handling) is necessary to recognise when pain relief is required. Post-acute phase, animals are generally monitored daily with less frequent detailed assessment. An example regime is assessment every other day from days 5 to 25 and twice a week from then, provided the animal's condition has stabilised. After the acute phase, animals should be weighed and body condition scored on assessment days.

\section{Suitable welfare indicators for mice and rats in RA studies}

The European Commission (EC) Expert Working Group on Severity Assessment guidance on recording clinical observations sets out 'high level' categories of observations, which are then broken down into areas to focus on when observing animals, then specific indicators to monitor within each area (European Commission 2012). Table 4 lists indicators from the EC guidance identified by Group members as most relevant to RA studies, categorised according to the EC system.

The EC has also published a worked example of this approach for Type II CIA in rats (European Commission 2013), and "Appendix" to this document sets out an example hypothetical 'score sheet' for mice using some of these indicators. Note that this is a generic example, not suitable for use without adaptation and tailoring to the species, strain and protocol. Some of the indicators and their applications are explained further below.

\section{Weight loss}

Animals on RA studies may lose weight, or weight may remain stable when an animal should be growing, either of which can be a concern. Scores are generally assigned for percentage weight loss. It is good practice to obtain the baseline weight for each animal and note the weight at which to implement the humane endpoint. In growing animals, it may be necessary to compare with age-matched controls.

Body condition scoring can be used to assess body fat and/or muscle mass loss (Ullman-Culleré and Foltz 1999), but this involves handling and palpating animals which may be painful. Discomfort can be reduced by using a clean piece of Vetbed ${ }^{\circledR}$ to restrain animals, or to rest them on during handling.

\section{Running wheels}

Reduced levels of running wheel use can infer levels of joint pain (Krug et al. 2009; Whittaker and Howarth 2014), but there is currently debate about running wheels as 'enrichment'. Some view them as providing additional activity, and in the experience of one author wheels help to reduce aggression. However, there are concerns that wheel running is an abnormal, and possibly addictive, behaviour because animals spend long periods running, sometimes to the detriment of other behaviours (Sherwin 1998; Würbel 2008; Richter et al. 2014). The utility of running wheels as an indicator of RA progression may justify providing them, provided that average group data are obtained rather than singly housing social animals to acquire individual data.

\section{'Pain faces'}

Research is ongoing into 'pain faces' in a number of species. A mouse grimace scale (MGS) and rat grimace scale (RGS), including elements such as 'orbital tightening' and 'nose bulge', have been developed for use when animals are experiencing acute pain (Langford et al. 2010; Sotocinal et al. 2011; Whittaker and Howarth 2014). These Grimace Scales can be useful for assessing animals in chronic pain if this also includes acute episodes. At one author's establishment the MGS is displayed in procedure rooms to help staff assess animals.

\section{Disturbed sleep}

Disturbed sleep has been noted in animals on RA studies (Andersen and Tufik 2000). It is unlikely that many facilities will have the resource to monitor sleep patterns at present, but non-invasive biotelemetry systems and behavioural monitoring software are both developing rapidly and may become more accessible. Such systems could also be used to monitor animals when active at night.

\section{Swollen paws}

Animals should be very gently caught and handled, and paws checked daily, from around day 14 following the initial induction (or from the point of inflammation). The digits and joints should be examined, and if there is swelling it should be noted how high up the limb this is present. Swelling may be measured with (preferably nonspring) callipers or by plethysmometry, as swollen paws are painful (Bolon et al. 2011) (Fig. 1). Swollen paws and 
Table 4 Useful indicators for welfare assessment of mice and rats used in RA studies

\begin{tabular}{|c|c|c|}
\hline $\begin{array}{l}\text { High level } \\
\text { category }\end{array}$ & Areas to focus on when observing animals & Specific indicators to monitor \\
\hline \multirow[t]{2}{*}{ Appearance } & $\begin{array}{l}\text { Body condition } \\
\text { Coat and skin condition }\end{array}$ & $\begin{array}{l}\text { Weight loss and/or loss of body condition } \\
\text { Ulceration } \\
\text { Faecal or urine staining } \\
\text { Unkempt or greasy coat } \\
\text { Scabbing, ulceration, infection at injection site associated with } \\
\text { adjuvant } \\
\text { Skin tenting (dehydration) } \\
\text { In adjuvant arthritis, crusting or lesions around eyes, ears, paws, tail; } \\
\text { tail 'ribbing' (changes in connective tissue) } \\
\text { Paw ulceration }\end{array}$ \\
\hline & $\begin{array}{l}\text { Discharge } \\
\text { Other }\end{array}$ & $\begin{array}{l}\text { Ocular discharge } \\
\text { 'Pain face', e.g., semi-closed eyes and nose bulge in mice }\end{array}$ \\
\hline Body functions & $\begin{array}{l}\text { Respiration } \\
\text { Food/water intake } \\
\text { Body temperature }\end{array}$ & $\begin{array}{l}\text { Dyspnoea or tachypnoea } \\
\text { Reduced } \\
\text { Decreased temperature, indicated by observing shivering or use of } \\
\text { thermography }\end{array}$ \\
\hline Environment & $\begin{array}{l}\text { Enclosure environment, including any litter, nesting } \\
\text { material, enrichment items }\end{array}$ & $\begin{array}{l}\text { Soft faeces or diarrhoea; or lack of faeces (constipation) } \\
\text { Poor quality nest } \\
\text { Reduced use of enrichment items such as chew blocks }\end{array}$ \\
\hline \multirow[t]{3}{*}{ Behaviours } & Social interaction & $\begin{array}{l}\text { Change in temperament or responsiveness. } \\
\text { Pain can have varying effects e.g., reduced aggression to conspecifics, } \\
\text { or increased aggression towards humans } \\
\text { Isolated or withdrawn from conspecifics }\end{array}$ \\
\hline & Posture and mobility & $\begin{array}{l}\text { Lethargy } \\
\text { Reduced wheel running }\end{array}$ \\
\hline & Other & $\begin{array}{l}\text { Vocalisation; spontaneous or invoked } \\
\text { Sleep disturbance } \\
\text { Less willing to take treats or to incorporate new material into nest }\end{array}$ \\
\hline $\begin{array}{l}\text { Procedure- } \\
\text { specific } \\
\text { indicators }\end{array}$ & $\begin{array}{l}\text { Indentified on the basis of the individual project, its } \\
\text { potential adverse effects and expected indicators of } \\
\text { these }\end{array}$ & $\begin{array}{l}\text { Abnormal gait (e.g., 'sledging' in severe cases) } \\
\text { Abnormal posture } \\
\text { Paw swelling } \\
\text { Clinical indicators e.g., data from von Frey tests, gait analysis } \\
\text { apparatus and software, data from imaging joints } \\
\text { Analgesia self-administration, where applicable } \\
\text { Serum biomarkers, if available as part of the project, could be used to } \\
\text { provide additional information about disease progression-but } \\
\text { blood samples should not be taken solely for this purpose }\end{array}$ \\
\hline $\begin{array}{l}\text { Free } \\
\quad \text { observations }\end{array}$ & $\begin{array}{l}\text { A severity assessment scheme should always include a } \\
\text { indicators of suffering }\end{array}$ & facility to note any observations of unexpected \\
\hline
\end{tabular}

Indicators in bold are especially relevant with regard to humane endpoints

digits may also be accompanied by reddening of the skin (Fig. 2).

An example approach to visually monitoring clinical scores of the hind paws in pristane adjuvant arthritic rats is outlined below (Table 5; Fig. 3). In this trial, all joints were assessed in order to determine an optimal scoring system relevant to that model, as different joints develop and resolve arthritis at different times.

In mice, distinguishing individual joints is difficult without handling animals and touching the paws. However, in the authors' experience an assessment system for mice in which whole paws are scored (Fig. 4) was as robust as a 
protocol that scored individual digits and joints (see "Appendix"). 'Global' scores for each paw, tailored to individual projects, can therefore be used for mice, avoiding handling.

Infrared thermography (using a video camera) has been suggested to monitor clinical severity, as foot temperature

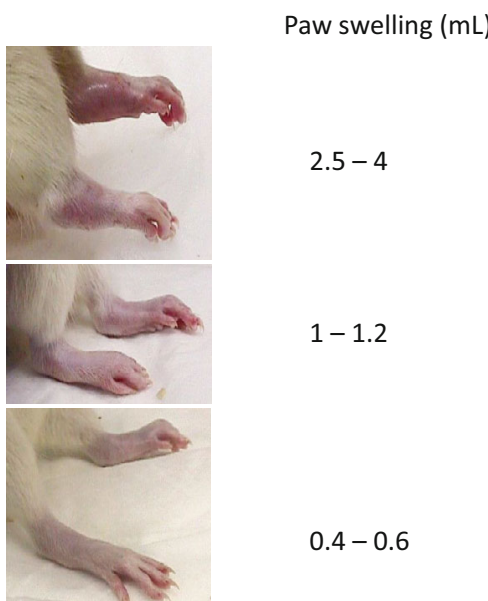

Fig. 1 Degrees of paw swelling in FCA adjuvant arthritic rats, measured using plethysmography. This illustrates significant, but well controlled, paw swelling to $2.5 \mathrm{~mL}$. Volumes above this are likely to cause severe pain and debilitation and should be considered a humane end point, as in the top paw. (From Bolon et al. (2011), reproduced by kind permission of Hindawi Publications Corp.) can be correlated with the degree of swelling (Jasemian et al. 2011). Thermographic images can be taken without anaesthesia, restraint or otherwise handling the animals, with welfare advantages if joints are painful.

\section{Self-administration of analgesics as a welfare indicator}

Analgesia self-administration can indicate discomfort or pain (Whittaker and Howarth 2014), either if self-administration is part of the project, or if pain relief can be provided during at least part of the study. It may be justifiable to conduct a pilot study using analgesic selfadministration to validate behavioural indicators of pain (Colpaert et al. 1980, 2001), even if analgesia cannot then be provided during the actual study.

\section{Humane endpoints}

'Humane endpoint' can be defined as 'the point at which an animal's pain and/or distress is terminated, minimised or reduced, by taking actions such as killing the animal humanely, terminating a painful procedure or giving treatment to relieve pain and/or distress' (see http://www. humane-endpoints.info).

The humane endpoints for each study will depend upon factors including its aims, the stage at which sufficient data are obtained, and sometimes whether a predetermined
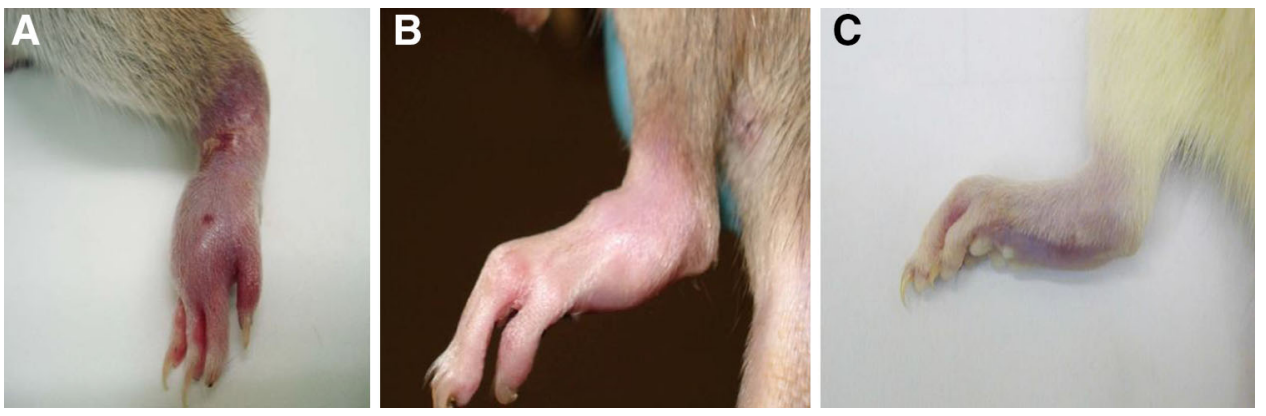

Fig. 2 Appearance of rat hind paws with arthritis following different doses of pristane. a Note swelling, redness and start of skin lesions. b Ankylosis at the chronic phase; histology shows active inflammation. The animals shown in $\mathbf{a}$ and $\mathbf{b}$ reached the humane endpoint and were humanely killed. $\mathbf{c}$ Well managed arthritis. The development of severe arthritis with lesions, as in $\mathbf{a}$, is not required as power can be maintained with lower doses of pristane as in $\mathbf{c}$, also reducing variability. (Courtesy M. Seed, University of East London)
Table 5 Example scoring scheme for investigating the pattern of paw involvement in pristane arthritis, using DA rats to determine outcomes for the full trial. This can be adapted for use with other models

\begin{tabular}{lcc}
\hline Arthritis score & Maximum points-hindpaws & Maximum points-forepaws \\
\hline 1 point for each swollen or red digit & 5 & 4 \\
1 point for each swollen knuckle & 5 & 4 \\
1 point for swollen midfoot & 1 & 1 \\
1 points for a swollen ankle/wrist & 1 & 1 \\
Total $(\times 2)$ & 24 & 20 \\
\hline
\end{tabular}



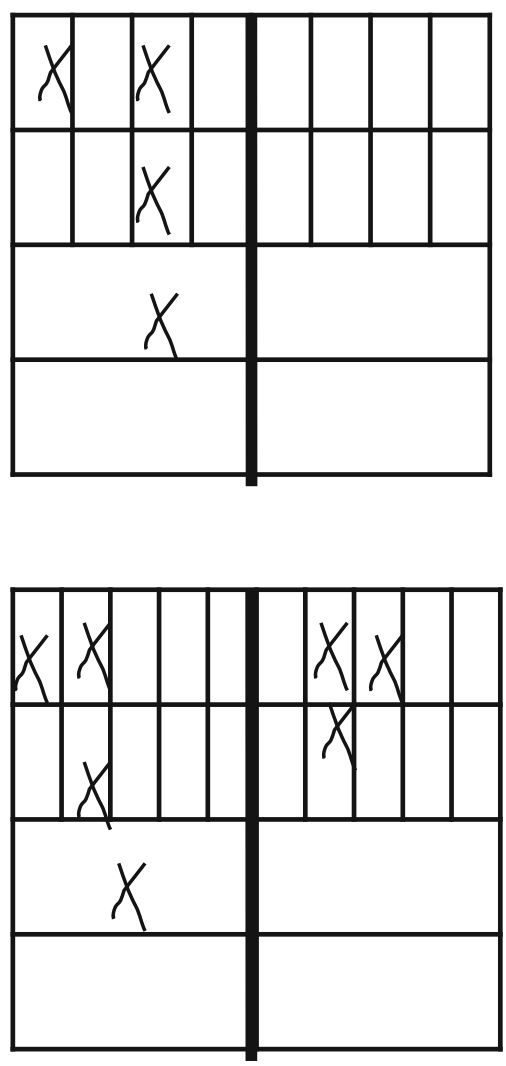

Fig. 3 System for recording paw scores. An example of a scoring system that can be used in pilot rodent trials to determine the pattern of disease expression and evaluate different scoring systems and determine power. The top rows represent the digits, second rows are the knuckles, and third and fourth rows are the midfoot and ankle/ wrist respectively. The scores in this case are: left front 4, right front 0, left hind 4, right hind 3 (courtesy M. Seed, University of East London)

'severity limit' has been reached. Local ethics or animal care and use committees may also have input into defining humane endpoints. However, the authors believe that there are some generally applicable limits regarding specific adverse effects in RA studies, at which point animals should be humanely killed. These are listed in Table 6 .
Other indicators are commonly used in combination to implement humane endpoints, but some involve subjective judgements and using several indicators requires careful consideration. A 'score sheet' approach, either noting whether indicators are present/absent or assigning numerical scores to these, can provide a useful adjunct to competent and empathetic human observers by helping to improve objectivity (see Sect. 6).

A number of judgements will need to be made including: the numerical score at which an animal should be humanely killed; whether one or more factors should be weighted; and whether and how duration should be taken into account (e.g., how long to maintain a medium/high scoring, but below threshold, animal). Issues like these should be discussed by the researcher, veterinarian, animal technologists and care staff, with appropriate input from the regulator and ethics or animal care and use committee.

\section{Experimental design and reduction}

Good experimental design is critical with respect to ensuring that projects are statistically robust, and that the correct number of animals is used to achieve the experimental objectives-neither too many, which causes avoidable suffering; nor too few, which is unethical if it means that animals are used in projects that have no significant benefit. For generic guidelines and principles to apply at the project planning stage, see Festing et al. (2002), Bate and Clark (2014) and the ARRIVE guidelines on reporting animal use (Kilkenny et al. 2010). Although ARRIVE primarily relates to writing up in vivo research, it is also a useful study design checklist and is available within the UK National Centre for the three Rs (NC3Rs) resource hub on experimental design; see http://www.nc3rs.org.uk/experimental-design.

In practice there are different, and sometimes competing, factors to take into account when determining appropriate numbers, including the experience of each individual
Fig. 4 Appearance of mouse front paws with progressively severe CIA arthritis. In this example scheme, $O$ normal, 1 digits swollen, 2 digits and pad swollen, 3 wrist/ankle, pad and digits swollen. (Courtesy Remi Okoye, Alex Vugler; UCB Celltech)

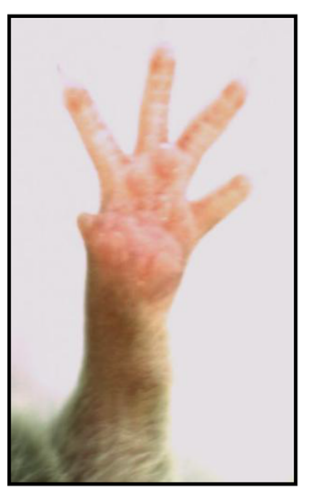

0

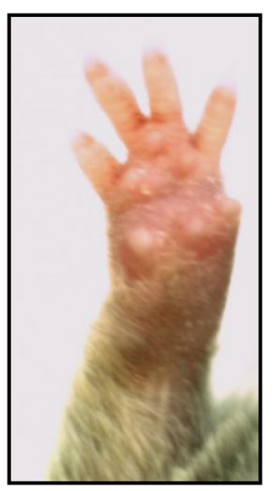

1

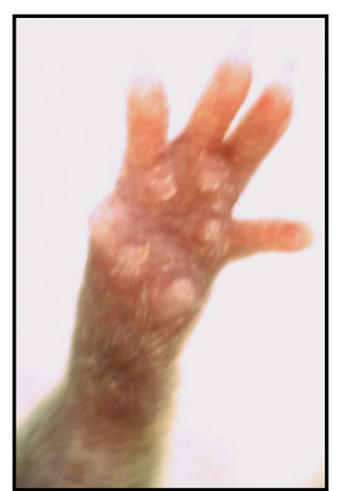

2

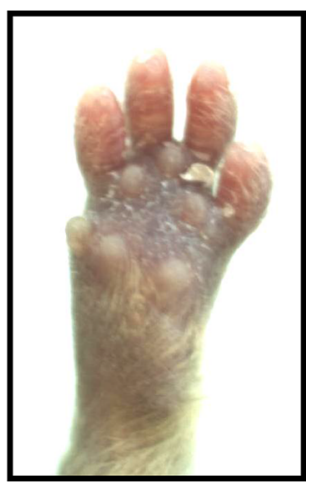

3 
Table 6 Humane endpoints for mice and rats in RA studies

\begin{tabular}{|c|c|}
\hline Adverse effect & Humane endpoints and comments \\
\hline $\begin{array}{l}\text { Ulceration may develop in induced models, but with good } \\
\text { practice this should only occur in a small proportion of mice } \\
\text { and rats }(<10 \% \text { in the authors' experience) }\end{array}$ & $\begin{array}{l}\text { The focus is generally not on diameter, but on whether there } \\
\text { are signs of healing, any secondary infection, ulcer depth, } \\
\text { whether the ulcer is wet, and behavioural signs of pain or } \\
\text { discomfort } \\
\text { However, if an ulcer diameter is }>5 \mathrm{~mm} \text {, the veterinarian or } \\
\text { senior animal technologist should be informed and consulted } \\
\text { about suitable treatment. The animal should be humanely } \\
\text { killed if there are no signs of healing within } 3 \text { days }\end{array}$ \\
\hline Abnormal gaits and postures & $\begin{array}{l}\text { 'Sledging', i.e., pushing the tail down to compensate for two } \\
\text { painful hind paws } \\
\text { Difficulty holding food } \\
\text { Prolonged }(>72 \mathrm{~h} \text { ) failure to weight bear on a limb }\end{array}$ \\
\hline $\begin{array}{l}\text { Severe paw swelling, assessed by visual scoring or using } \\
\text { callipers or plethysmography (Figs. } 1,2,4 \text { ) } \\
\text { Paw size increases may need to be corrected for growth }\end{array}$ & $\begin{array}{l}\text { The researcher, animal technologists and veterinarian should } \\
\text { collaborate to define the maximum level of swelling, the } \\
\text { number of paws that may be affected, how this will be } \\
\text { assessed, and how long severe swelling should be permitted } \\
\text { to continue } \\
\text { Humane endpoints should always be implemented if swelling } \\
\text { forces the digits to splay, or encompasses the entire foot and } \\
\text { ankle, beginning to rise up the lower leg }\end{array}$ \\
\hline $\begin{array}{l}\text { Spontaneous vocalisation or squeaking and quivering when } \\
\text { picked up or handled }\end{array}$ & $\begin{array}{l}\text { Rodents generally vocalise at ultrasonic frequencies, so audible } \\
\text { calls can indicate severe pain or distress }\end{array}$ \\
\hline $\begin{array}{l}\text { Arthritic rats can also vocalise in the cage when jostling with } \\
\text { cagemates }\end{array}$ & $\begin{array}{l}\text { Absence of vocalisation does not indicate acceptable pain } \\
\text { levels, as clinical scores can reach humane end points } \\
\text { without audible vocalisation }\end{array}$ \\
\hline $\begin{array}{l}\text { Weight loss } \\
\text { Exceptions can be made for treatments expected to induce } \\
\text { weight loss, such as glucocorticoids }\end{array}$ & $\begin{array}{l}20 \% \text { is generally used in RA studies, or some protocols factor } \\
\text { in the duration, e.g., endpoint of } 15 \% \text { loss that does not } \\
\text { begin to reverse within } 5 \text { days } \\
\text { Condition scoring can also be used, with scores that indicate } \\
\text { humane endpoints of additional food and/or hydration } \\
\text { support or humane killing }\end{array}$ \\
\hline $\begin{array}{l}\text { Study-specific end points } \\
\text { These involve an additional pain or anaesthesia burden, so } \\
\text { should be used only if data are available as part of the } \\
\text { scientific output of the study }\end{array}$ & $\begin{array}{l}\text { Peripheral blood biomarkers may be used as earlier primary } \\
\text { indicators of the inflammatory response, rather than gross } \\
\text { inflammatory load, e.g., acute phase proteins or urinary } \\
\text { cartilage breakdown products might be present before } \\
\text { clinical deterioration and suffering }\end{array}$ \\
\hline & $\begin{array}{l}\text { Data from imaging joints using X-ray or micro-computer } \\
\text { tomography }(\mu \mathrm{CT}) \text {, e.g., erosion and periostitis endpoints }\end{array}$ \\
\hline
\end{tabular}

animal. For example, it is sometimes necessary to balance welfare against group sizes, but the authors believe that welfare considerations should predominate. That is, it can be preferable in principle to use more animals, with less suffering to each individual (or better welfare), provided that scientific integrity is not compromised.

\section{Reporting animal use in RA studies}

Literature reviews have identified serious issues with the design, analysis and reporting of animal use in a significant number of publications (Kilkenny et al.
2009; Baker et al. 2014; Bara and Joffe 2014; Moja et al. 2014). The authors support the ARRIVE guidelines and believe that it is critically important to include information on efforts made to replace animals, reduce their use and suffering and improve their welfare in materials and methods sections, or as supplementary materials depending on the journal's approach and policy (Osborne et al. 2009). Posters and talks can also include brief information on the three $\mathrm{Rs}$, or supplementary information can be included on flyers to accompany poster presentations. This will help to disseminate good practice and enable proper interpretation of the results. 
Table 7 List of key recommendations to help refine the use of mice and rats in RA studies

\begin{tabular}{|c|c|}
\hline Recommendation & Sections \\
\hline Review the sources of variation listed in Table 1 and ensure that each is addressed & 2.3 \\
\hline $\begin{array}{l}\text { Set out the whole life experience of each animal and consider how each potentially painful or distressing event could be } \\
\text { refined, using Table } 2\end{array}$ & 3 \\
\hline Use the list of principles in Sect. 3 to review housing and husbandry, addressing any omissions & 3 \\
\hline $\begin{array}{l}\text { Critically question any statements that environmental enrichment has a negative impact on data quality; ask for empirical } \\
\text { evidence and be prepared to conduct or permit pilot studies if appropriate }\end{array}$ & 3 \\
\hline If using an inducer, review its nature, formulation and administration protocol & 3 \\
\hline If using LPS or CFA, review the justification and necessity and ensure appropriate refinements and humane endpoints & 4 \\
\hline $\begin{array}{l}\text { Review criteria for humane endpoints regarding 'maximum end point responses' and 'therapeutic dosing regimes'. If } \\
\text { responsible for designing projects, suggest the topic for discussion by the ethics or animal care and use committee }\end{array}$ & 4 \\
\hline $\begin{array}{l}\text { For studies within the chronic resolving phase, critically review the model, the potential to reduce acute phase severity and } \\
\text { duration, welfare assessment protocols and humane endpoints }\end{array}$ & 4 \\
\hline $\begin{array}{l}\text { Review the justification and necessity for control groups, sharing these wherever possible without compromising the science, } \\
\text { and refining humane endpoints }\end{array}$ & 4,7 \\
\hline $\begin{array}{l}\text { Do not assume that analgesia will negatively affect data. Use the literature, and undertake or permit pilot studies as necessary, } \\
\text { to evaluate the effects of analgesia on both welfare and science }\end{array}$ & 5 \\
\hline Carefully consider how to administer analgesia, including pros and cons of gavage, parenteral- and self-administration & 5.4 \\
\hline $\begin{array}{l}\text { Keep up with developments in animal monitoring technology, e.g., new software, activity meters and thermography-avoiding } \\
\text { those that require single housing }\end{array}$ & 6 \\
\hline $\begin{array}{l}\text { Ensure that appropriate welfare assessment protocols are defined, and regularly reviewed, with a variety of inputs including the } \\
\text { veterinarian, researchers, animal technologists and the ethics or animal care and use committee }\end{array}$ & 6 \\
\hline $\begin{array}{l}\text { Implement the ' } R \text { ' of reduction thoughtfully, ensuring that sufficient power is maintained while minimising numbers and } \\
\text { severity }\end{array}$ & 8 \\
\hline Use the ARRIVE guidelines as a checklist when designing projects as well as when writing papers for publication & 9 \\
\hline
\end{tabular}

\section{Recommendations for the future of $\mathrm{RA}$ research using animals}

Future work should aim to further reduce lifetime severity in RA studies, whilst maintaining or even improving opportunities for medical advances. This would be achieved through the following:

- Further research into the use of analgesia, with respect to suitable agents, effects on welfare and the science, timing of administration and self-administration.

- A systematic review of the provision of analgesia in arthritis experiments.

- Improved indicators of pain and distress, such as accessible computer-assisted behavioural analysis.

- Less severe models, e.g., not requiring the use of potentially severe inducers such as CFA.

- More physiologically relevant spontaneous models using GA mice, which will decrease the number of procedures because it will not be necessary to induce RA.

- International guidelines for refined experimental protocols, including humane endpoints.

- Better sharing and publication of all three Rs in RA studies.
- Greater support for the development and uptake of in vitro, in silico and epidemiological approaches to RA research.

\section{Conclusion}

The Working Group believes that there is considerable scope to reduce the suffering and improve the welfare of mice and rats in RA studies, and hopes that this resource will support and encourage ongoing efforts towards this important goal. Table 7 sets out some key recommendations taken from the text, which can be used as a check list when designing, conducting or reviewing projects, to help reduce severity and ensure that appropriate refinements have been implemented wherever possible.

Acknowledgments The authors would like to thank Cancer Research UK and the British Pharmacological Society for providing meeting rooms and facilities for Working Group meetings. We also thank Dr Michael Burnet, Synovo GmbH, Tübingen, Germany, for unpublished information on the use of opiates in RA models. ROW has received grant support from the Kennedy Trust for Rheumatology Research and the European Union (FP7 Project PRIAT, Grant No. 305309). 


\section{Compliance with Ethical Standards}

Conflict of interest The authors declare that they have no competing interests.

Open Access This article is distributed under the terms of the Creative Commons Attribution 4.0 International License (http:// creativecommons.org/licenses/by/4.0/), which permits unrestricted use, distribution, and reproduction in any medium, provided you give appropriate credit to the original author(s) and the source, provide a link to the Creative Commons license, and indicate if changes were made.

\section{Appendix}

See Table 8.

Table 8 Generic example of a score sheet for mice used in rheumatoid arthritis studies

\begin{tabular}{|c|c|c|c|c|c|}
\hline \multicolumn{6}{|l|}{ Initial body weight (g) } \\
\hline \multicolumn{6}{|l|}{$\begin{array}{l}\text { Minimum weight }(\mathrm{g}) \\
=0.8 \times \text { initial }\end{array}$} \\
\hline & Day & 1 & 2 & 3 & 4 \\
\hline \multicolumn{6}{|l|}{ Body weight } \\
\hline $0-5 \%$ & 0 & & & & \\
\hline $5-10 \%$ & 1 & & & & \\
\hline $10-15 \%$ & 2 & & & & \\
\hline$\geq 15 \%$ for up to 5 days $^{a}$ & 4 & & & & \\
\hline $20 \%$ & HEP & & & & \\
\hline \multicolumn{6}{|l|}{ Coat condition } \\
\hline Normal & 0 & & & & \\
\hline Lack of grooming & 1 & & & & \\
\hline Staring coat & 2 & & & & \\
\hline \multicolumn{6}{|l|}{ Mouse Grimace Scale } \\
\hline Not present & 0 & & & & \\
\hline Moderate & 1 & & & & \\
\hline Severe & 2 & & & & \\
\hline Present for $24 \mathrm{~h}$ & HEP & & & & \\
\hline \multicolumn{6}{|l|}{ Behaviour } \\
\hline Isolated from cagemates & 5 & & & & \\
\hline \multicolumn{6}{|c|}{ Arthritic paw score (see table below) } \\
\hline Normal & 0 & & & & \\
\hline Total 1 - 3 & 2 & & & & \\
\hline Total 4-6 & 4 & & & & \\
\hline Total 7-9 & 8 & & & & \\
\hline Total 10 or more & HEP & & & & \\
\hline Skin discolouration ${ }^{b}$ & 6 & & & & \\
\hline \multicolumn{6}{|l|}{ Total score } \\
\hline $\begin{array}{l}\text { Free text box for addition } \\
\text { observations }\end{array}$ & & & & & \\
\hline
\end{tabular}

Paw scoring system: $0=$ normal, 1 = digits swollen, 2 = digits and pad swollen, $3=$ wrist $/$ ankle, pad and digits swollen

\begin{tabular}{|l|l|l|l|l|l|}
\hline Day & $0-3$ & & & & \\
\hline Right front & $0-3$ & & & & \\
\hline Left front & $0-3$ & & & & \\
\hline Right back & $0-3$ & & & & \\
\hline Left back & & & & \\
\hline Total & & & & \\
\hline
\end{tabular}

a If animals have lost $15 \%$ or more of their body weight, weigh them daily. Euthanase if no recovery is seen for 4 days or weight loss reaches $20 \%$.

b Skin discolouration-an area of skin on the paw with a noticeably different colour, e.g., redness where swelling has stretched the skin (Fig. 2) HEP = humane endpoint

Actions:

\begin{tabular}{|l|l|}
\hline 0 & Normal, no action required \\
\hline $1-5$ & Disease evident, monitor carefully \\
\hline $6-10$ & Implement pain management protocols \\
\hline $11-13$ & $\begin{array}{l}\text { Seek advice from senior animal technologist or veterinarian; consider humane killing; if no senior animal technologist or } \\
\text { veterinarian is available then euthanase the animal }\end{array}$ \\
\hline 14 & HEP \\
\hline
\end{tabular}

This should be tailored to meet the characteristics and requirements of individual projects 


\section{References}

Abelson KSP, Jacobsen KR, Sundbom R, Kalliokoski O, Hau J (2012) Voluntary ingestion of nut paste for administration of buprenorphine in rats and mice. Lab Anim 46:349-351

Andersen ML, Tufik S (2000) Altered sleep and behavioural patterns of arthritic rats. Sleep Res Online 3:161-167

Baker D, Lidster K, Sottomayor A, Amor S (2014) Two years later: journals are not yet enforcing the ARRIVE guidelines on reporting standards for pre-clinical animal studies. PLoS Biol 12(1):e1001756

Bara M, Joffe AR (2014) The ethical dimension in published animal research in critical care: the public face of science. Crit Care 18:R15

Bas DB, Su J, Sandor K, Agalave NM, Lundberg J, Codeluppi S, Baharpoor A, Nandakumar KS, Holmdahl R, Svensson CI (2012) Collagen antibody-induced arthritis evokes persistent pain with spinal glial involvement and transient prostaglandin dependency. Arthritis Rheum 64:3886-3996

Bate ST, Clark RA (2014) The design and statistical analysis of animal experiments. Cambridge University Press, Cambridge

Baumans V, Brain PF, Brugere H, Clausing P, Jeneskog T, Perretta G (1994) Pain and distress in laboratory Rodents and Lagomorphs: FELASA Report No2. Lab Anim 28:97-112

Bevaart L, Vervoordeldonk MJ, Tak PP (2010) Evaluation of therapeutic targets in animal models of arthritis. Arthritis Rheum 62:2192-2205

Bianchi M, Panerai AE (1996) The dose-related effects of paracetamol on hyperalgesia and nociception in the rat. Br J Pharmacol 117:130-132

Bolon B, Stolina M, King C, Middleton S, Gasser J, Zack D, Feige U (2011) Rodent preclinical models for developing novel antiarthritic molecules: comparative biology and preferred methods for evaluating efficiency. Journal of Biomedicine and Biotechnology. doi:10.1155/2011/569068

Brand DD, Latham KA, Rosloniec EF (2007) Collagen-induced arthritis. Nat Protoc 2:1269-1275

Carbone L (2011) Pain in laboratory animals: the ethical and regulatory imperatives. PLoS One 6(9):e21578

Chan SC, Chan CC, Kwan AS, Ting KH, Chui TY (2012) Orienting attention modulates pain perception: an ERP study. PLoS One 7(6):e40215

Christianson CA, Corr M, Firestein GS, Mobargha A, Yaksh TL, Svensson CI (2010) Characterization of the acute and persistent pain state present in $\mathrm{K} / \mathrm{BxN}$ serum transfer arthritis. Pain 151:394-403

Colpaert FC (1987) Evidence that adjuvant arthritis in the rat is associated with chronic pain. Pain 28:201-222

Colpaert FC, De Witte Ph, Maroli AN, Awouters F, Niemegeers CJE, Janssen PAJ (1980) Self-administration of the analgesic suprofen in arthritic rats: evidence of mycobacterium butyricum induced arthritis as an experimental model of chronic pain. Life Sci 27:921-928

Colpaert FC, Tarayre JP, Alliaga M, Bruins Slot LA, Attal N, Koek W (2001) Opiate self-administration as a measure of chronic nociceptive pain in arthritic rats. Pain 91:33-45

Commission European (2010) Directive 2010/63/EU of the European Parliament and of the Council of 22 September 2010 on the protection of animals used for scientific purposes. Off $\mathrm{J}$ Eur Union L276:33-79

Dinda A, Gitman M, Singhal PC (2005) Immunomodulatory effect of morphine: therapeutic implications. Expert Opin Drug Saf 4:669-675
Earl JR, Claxson AW, Blake DR, Morris CJ (1994) Proinflammatory effects of morphine in the rat adjuvant arthritis model. Int $\mathbf{J}$ Tissue React 16:163-170

European Commission (2012) Working document on a severity assessment framework. http://ec.europa.eu/environment/ chemicals/lab_animals/pdf/Endorsed_Severity_Assessment.pdf. Accessed 5 May 2015

European Commission (2013) Examples to illustrate the process of severity classification, day-to-day assessment and actual severity assessment. http://ec.europa.eu/environment/chemicals/lab_ animals/pdf/examples.pdf. Accessed 5 May 2015

Festing MW, Overend P, Das RG, Borja MC, Berdoy M (2002) The design of animal experiments: reducing the use of animals in research through better experimental design; laboratory animal handbooks No 14. R Soc Med Press, London

Ford GK, Moriarty O, Mc Guire BE, Finn DP (2008) Investigating the effects of distracting stimuli on nociceptive behaviour and associated alterations in brain monoamines in rats. Eur $\mathrm{J}$ Pain 12:970-979

Gaskill BN, Rohr S, Pajor EA, Lucas JR, Garner JP (2009) Some like it hot: mouse temperature preferences in laboratory housing. Appl Anim Behav Sci 116:279-285

Gaskill BN, Gordon CJ, Pajor EA, Lucas JR, Davis JK, Garner JP (2012) Heat or insulation: behavioral titration of mouse preference for warmth or access to a nest. PLoS One 7(3):e32799

Gentle MJ, Tilston VL (1999) Reduction in peripheral inflammation by changes in attention. Physiol Behav 66:289-292

Gouveia K, Hurst J (2013) Reducing mouse anxiety during handling: effect of experience with handling tunnels. PLoS One 8(6):e66401

Havey J, Vlasses FR, Vlasses PH, Ludwig-Beymer P, Hackbarth D (2014) The effect of animal-assisted therapy on pain medication use after joint replacement. Anthrozoös 27:361-369

Hawkins P, Morton DB, Burman O, Dennison N, Honess P, Jennings M, Lane S, Middleton V, Roughan JV, Wells S, Westwood K (2011) A guide to defining and implementing protocols for the welfare assessment of laboratory animals. Lab Anim 45:1-13

Honoré P, Menning PM, Rogers SD, Nichols ML, Basbaum AI, Besson J-M, Mantyh PW (1999) Spinal substance P receptor expression and internalization in acute, short-term, and long-term inflammatory pain states. J Neurosci 19:76707678

Hurst JL, West R (2010) Taming anxiety in mice by non-aversive handling. Nat Methods 7:825-826. doi:10.1038/nmeth.1500

Hutchinson E, Avery A, VandeWoude S (2005) Environmental enrichment for laboratory rodents. ILAR J 46:148-161

Jacobsen KR, Kalliokoski O, Hau J, Abelson KSP (2011) Voluntary ingestion of buprenorphine in mice. Anim Welf 20:591-596

Jasemian Y, Svendsen P, Deleuran B, Dagnaes-Hansen F (2011) Refinement of the collagen induced arthritis model in rats by infrared thermography. Br J Med Med Res 1:469-477

Julià A, Erra A, Palacio C, Tomas C, Sans X, Barceló P, Marsal S (2009) An eight-gene blood expression profile predicts the response to infliximab in rheumatoid arthritis. PLoS One 4(10):e7556

Karp CL (2012) Unstressing intemperate models: how cold stress undermines mouse modelling. J Exp Med 209:1069-1074

Khachigian LM (2006) Collagen antibody-induced arthritis. Nat Protoc 1(5):2512-2516

Kilkenny C, Parsons N, Kadyszewski E, Festing MWF, Cuthill IC, Fry D, Hutton J, Altman DG (2009) Survey of the quality of experimental design, statistical analysis and reporting of research using animals. PLoS One 4:e7284 
Kilkenny C, Browne WJ, Cuthill IC, Emerson M, Altman DG (2010) Improving bioscience research reporting: the ARRIVE guidelines for reporting animal research. PLoS Biol 8(6):e1000412

Kokolus KM, Capitano ML, Lee CT, Eng JW, Waight JD, Hylander BL, Sexton S, Hong CC, Gordon CJ, Abrams SI, Repasky EA (2013) Baseline tumor growth and immune control in laboratory mice are significantly influenced by subthermoneutral housing temperature. PNAS 110:20176-20181

Kollias G, Papadaki P, Apparailly F, Vervoordeldonk MJ, Holmdahl R, Baumans V, Desaintes C, Di Santo J, Distler J, Garside P, Hegen M, Huizinga TWJ, Jüngel A, Klareskog L, McInnes I, Ragoussis I, Schett G, Hart B, Tak PP, Toes R, van den Berg WB, Wurst W, Gay S (2011) Animal models for arthritis: innovative tools for prevention and treatment. Ann Rheum Dis 70:1357-1362

Krug HE, Frizelle S, McGarraugh P, Mahowald ML (2009) Pain behavior measures to quantitate joint pain and response to neurotoxin treatment in murine models of arthritis. Pain Med 10:1218-1228

Langford DJ, Bailey AL, Chanda ML, Clarke SE, Drummond TE, Echols S, Glick S, Ingrao J, Klassen-Ross T, LaCroix-Fralish ML, Matsumiya M, Sorge RE, Sotocinal SG, Tabaka JM, Wong D, van den Maagdenberg AMJM, Ferrari MD, Craig KD, Mogil JS (2010) Coding of facial expressions of pain in the laboratory mouse. Nat Methods 7:447-449

Livingston A, Chambers P (2000) The physiology of pain. In: Flecknell P, Waterman-Pearson A (eds) Pain management in animals. WB Saunders \& Harcourt, London, pp 9-19

Maicas N, Ibáñez L, Alcaraz MJ, Úbeda A, Ferrándiz ML (2012) Prostaglandin D2 regulates joint inflammation and destruction in murine collagen-induced arthritis. Arthritis Rheum 64(1):130-140

Malik N, Burnet M, Jones R, Schett G, Seed M (2011) Differential joint disease expression in pristane-induced arthritis-a systematic profile of disease progression in Da/Olahsd rats. Inflamm Res 60:186

Marashi V, Barnekow A, Ossendorf E, Sachser N (2003) Effects of different forms of environmental enrichment on behavioral, endocrinological, and immunological parameters in male mice. Horm Behav 43:281-292

McCarthy R, Goess C, Rundell L, Cuff C, Prisca H, Andrew L (2012) Pain and inflammation coincide in a collagen-induced arthritis model in rats. J Immunol 188(177): 17

McGonigle P, Ruggeri B (2014) Animal models of human disease: challenges in enabling translation. Biochem Pharmacol $87: 162-171$

McQueen DS, Iggo A, Birrell GJ, Grubb BD (1991) Effects of paracetamol and aspirin on neural activity of joint mechanonociceptors in adjuvant arthritis. Br J Pharmacol 104:178-182

Mikkelsen LF, Sørensen DB, Krohn T, Lauritzen B, Dragsted N, Hansen AK, Ottesen JL (2010) Clinical pathology and cardiovascular parameters are not influenced by housing rats under increased environmental complexity. Anim Welf 19:449-460

Moja L, Pecoraro V, Ciccolallo L, Dall'Olmo L, Virgili G, Garattini S (2014) Flaws in animal studies exploring statins and impact on meta-analysis. Eur J Clin Invest. doi:10.1111/eci.12264

Molina-Cimadevila MJ, Segura S, Merino C, Ruiz-Reig N, Andrés B, de Madaria E (2014) Oral self-administration of buprenorphine in the diet for analgesia in mice. Lab Anim 48:216-224

Montero-Melendez T, Patel HB, Seed M, Nielsen S, Jonassen TE, Perretti M (2011) The melanocortin agonist AP214 exerts antiinflammatory and proresolving properties. Am J Pathol 179:259-269

National Research Council (2011) Guide for the care and use of laboratory animals, 8th edn. National Academies Press, Washington, DC
Neidhart M, Gay RE, Gay S (2000) Anti-interleukin-1 and anti-CD44 interventions producing significant inhibition of cartilage destruction in an in vitro model of cartilage invasion by rheumatoid arthritis synovial fibroblasts. Arthritis Rheum 43:1719-1728

NHMRC (National Health and Medical Research Council) (2008) The assessment and alleviation of pain and distress in research animals. In: Guidelines to promote the wellbeing of animals used for scientific purposes. National Health and Medical Research Council. Australian Government. http://www.nhmrc.gov.au/ index.htm. Accessed 5 May 2015

Osborne NJ, Payne D, Newman ML (2009) Journal editorial policies, animal welfare and the 3Rs. Am J Bioeth 9:55-59

Paska W, McDonald KJ, Croft M (1986) Studies on type-II collageninduced arthritis in mice. Agents Actions 18:413-420

Patel HB, Dawson B, Humby F, Blades M, Pitzalis C, Burnet M, Seed M (2010) Animal models of rheumatoid arthritis. In: Serhan CN, Ward PA, Gilroy DW (eds) Fundamentals of inflammation. Cambridge University Press, Cambridge, pp 385-412

Peck Y, Wang D-A (2013) Three-dimensionally engineered biomimetic tissue models for in vitro drug evaluation: delivery, efficacy and toxicity. Expert Opin Drug Deliv 10:369-383

Pretzel D, Pohlers D, Weinert S, Kinne RW (2009) In vitro model for the analysis of synovial fibroblast-mediated degradation of intact cartilage. Arthritis Res Ther 11:R25. doi:10.1186/ar2618

Pulichino AM, Rowland S, Wu T, Clark P, Xu D, Mathieu MC, Riendeau D, Audoly LP (2006) Prostacyclin antagonism reduced pain and inflammation in rodent models of hyperalgesia and chronic arthritis. J Pharmacol Exp Ther 319:1043-1050

Ren K, Dubner R (2010) Interactions between the immune and nervous systems in pain. Nat Med 16:1267-1276

Ribel-Madsen S, Bartels EM, Stockmarr A, Borgwardt A, Cornett C, Danneskiold-Samsøe B, Vincent TL, Williams RO, Maciewicz R, Silman A, Garside P (2012) Mapping pathogenesis of arthritis through small animal models. Rheumatology 51:1931-1941

Richter SH, Gass P, Fuss J (2014) Resting is rusting: a critical view on rodent wheel-running behavior. Neuroscientist 20:313-325

Sherwin CM (1998) Voluntary wheel running: a review and novel interpretation. Anim Behav 56:11-27

Smolian H, Thiele S, Kolkenbrock H, Zacher J, Aicher W, Schultz O, Burmester GR, Sittinger M (2001) Establishment of an in vitro model for rheumatoid arthritis as test system for therapeutical substances. ALTEX 18:265-280

Sotocinal SG, Sorge RE, Zaloum A, Tuttle AH, Martin LJ, Wieskopf JS, Mapplebeck JCS, Wei P, Zhan S, Zhang S, McDougall JJ, King OD, Mogil JS (2011) The rat grimace scale: a partially automated method for quantifying pain in the laboratory rat via facial expressions. Mol Pain 7:55. doi:10.1186/1744-8069-7-55

Strand V, Kimberly R, Isaacs JD (2007) Biologic therapies in rheumatology: lessons learned, future directions. Nat Rev Drug Discov 6:75-92

Tischkau SA, Mukai M (2009) Activation of aryl hydrocarbon receptor signalling by cotton balls used for environmental enrichment. JAALAS 48:357-362

Trivedi SG, Newson J, Rajakariar R, Jacques TS, Hannon R, Kanaoka Y, Equchi N, Colville-Nash P, Gilroy DW (2006) Essential role for hematopoietic prostaglandin D2 synthase in the control of delayed type hypersensitivity. Proc Natl Acad Sci USA 103:5179-5184

Ullman-Culleré MH, Foltz CJ (1999) Body condition scoring: a rapid and accurate method for assessing health status in mice. Lab Anim Sci 49:319-323

Ulrich RS (1984) View through a window may influence recovery from surgery. Science $224: 420-421$ 
van der Worp HB, Howells DW, Sena ES, Porritt MJ, Rewell S, O'Collins V, MacLeod MR (2010) Can animal models of disease reliably inform human studies? PLoS Med 7(3):e1000245

Van Loo P, Hauet F, Wagenaar J, Hellebrekers L, van Eden W, Baumans V (2006) Alleviation of pain and discomfort in adjuvant arthritis rats. In Report of the 2005 RSPCA/UFAW Rodent Welfare Meeting, edited by P Hawkins. Lab Anim 35:29-38

Vincent TL, Williams RO, Maciewicz R, Silman A, Garside P (2012) Mapping pathogenesis of arthritis through small animal models. Rheumatol 51:1931-1941

Volker D, Bate M, Gentle R, Garg M (2000) Oral buprenorphine is anti-inflammatory and modulates the pathogenesis of streptococcal cell wall polymer-induced arthritis in the Lew/SSN rat. Lab Anim 34:423-429

Whittaker AL, Howarth GS (2014) Use of spontaneous behaviour measures to assess pain in laboratory rats and mice: how are we progressing? Appl Anim Behav Sci 151:1-12
Wooley PH, Luthra HS, Stuart JM, David CS (1981) Type II collagen-induced arthritis in mice. I. Major histocompatibility complex (I region) linkage and antibody correlates. J Exp Med 154:688-700

Würbel H (2008) The motivational basis of caged rodents' stereotypies. In: stereotypic animal behaviour: fundamentals and applications to welfare, 2nd edn. CAB International, Wallingford, pp 86-120

Yoshitomi H, Sakaguchi N, Kobayashi K, Brown GD, Tagami T, Sakihama T, Hirota K, Tanaka S, Nomura T, Miki I, Gordon S, Akira S, Nakamura T, Sakaguchi S (2005) A role for fungal $\beta$ glucans and their receptor Dectin-1 in the induction of autoimmune arthritis in genetically susceptible mice. J Exp Med 201:949-960 